\title{
Fully integrating pathophysiological insights in COPD: an updated working disease model to broaden therapeutic vision
}

\author{
E. Haydn Walters ${ }^{1}$, Shakti D. Shukla $\mathbb{1}^{2,3}$, Malik Q. Mahmood ${ }^{4}$ and Chris Ward ${ }^{5}$
}

${ }^{1}$ School of Medicine and Menzies Institute, University of Tasmania, Hobart, Australia. ${ }^{2}$ Priority Research Centre for Healthy Lungs and School of Medicine and Public Health, Faculty of Health and Medicine, University of Newcastle, Callaghan, Australia. ${ }^{3}$ Hunter Medical Research Institute, New Lambton Heights, Australia. ${ }^{4}$ School of Medicine, Faculty of Health, Deakin University, Waurn Ponds, Australia. ${ }^{5}$ Translational and Clinical Research Institute, Faculty of Medical Sciences, Newcastle University Medical School, Newcastle University, UK.

Corresponding author: E. Haydn Walters (Haydn.Walters@utas.edu.au)

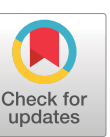

This version is distributed under the terms of the Creative Commons Attribution NonCommercial Licence 4.0. For commercial reproduction rights and permissions contact permissions@ersnet.org

Received: 16 Nov 2020 Accepted: 18 Feb 2021

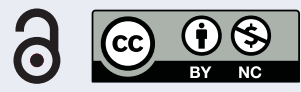

Shareable abstract (@ERSpublications)

We discuss the pathogenesis and pathophysiology of COPD, emphasising their need to be reassessed as a whole and integrated with traditional concepts to refine the disease paradigm. This is urgently needed to open-up thinking about therapeutic targets. https://bit.ly/3pTyrsi

Cite this article as: Walters EH, Shukla SD, Mahmood MQ, et al. Fully integrating pathophysiological insights in COPD: an updated working disease model to broaden therapeutic vision. Eur Respir Rev 2021; 30: 200364 [DOI: 10.1183/16000617.0364-2020].

\section{Abstract}

Our starting point is that relatively new findings into the pathogenesis and pathophysiology of airway disease in smokers that lead to chronic obstructive pulmonary disease (COPD) need to be reassessed as a whole and integrated into "mainstream" thinking along with traditional concepts which have stood the test of time. Such a refining of the accepted disease paradigm is urgently needed as thinking on therapeutic targets is currently under active reconsideration. We feel that generalised airway wall "inflammation" is unduly over-emphasised, and highlight the patchy and variable nature of the pathology (with the core being airway remodelling). In addition, we present evidence for airway wall disease in smokers/COPD as including a hypocellular, hypovascular, destructive, fibrotic pathology, with a likely spectrum of epithelialmesenchymal transition states as significant drivers of this remodelling. Furthermore, we present data from a number of research modalities and integrate this with the aetiology of lung cancer, the role of chronic airway luminal colonisation/infection by a specific group of "respiratory" bacteria in smokers (which results in luminal inflammation) and the central role for oxidative stress on the epithelium. We suggest translation of these insights into more focus on asymptomatic smokers and early COPD, with the potential for fresh preventive and therapeutic approaches.

\section{Introduction}

The authoritative source of advice and information on chronic obstructive pulmonary disease (COPD) internationally over the past 20 years has been the Global Initiative for Chronic Obstructive Lung Disease (GOLD). This was set up in 1998 with cooperation by the National Heart, Lung, and Blood Institute (NHLBI) of the US National Institutes of Health (NIH) and the World Health Organization (WHO). It has produced further comprehensive reports in 2001, 2006, 2011 and 2017-2020 [1-4]. This has transformed the COPD landscape in a positive way, with one considerable achievement being the promotion of clinical research activity leading to attempts at tailored management plans. However, there are now calls to revise the conventional prevailing approaches to this important disease, emphasising more the potential benefits and opportunities arising from anticipating the diagnosis even before classical COPD is present and enabling the treatment of COPD and even "pre-COPD” at an early stage [5-7]. We sympathise with these authors and have written this article to stimulate further positive discussion. We also build on these calls by suggesting our understanding of underlying disease processes needs to be updated to inform this discussion and future priorities. Indeed, others have recently emphasised the urgent need for this and indicated airway remodelling as a potential central issue [7]. Our intent is to systematise the balance of evidence for such a primary remodelling disease, its likely mechanisms (including interactions with airway 
luminal pathology) and the implications for disease management before COPD ensues, even and perhaps especially in smokers.

The airway inflammation hypothesis of COPD airways

A key success of initiatives such as GOLD and COPDGene has been in promoting the assessment of clinical trajectory and severity in overt disease, emphasising airway function and more recently symptoms and acute exacerbations (AEs). Sections on pathogenesis have always tended to be less central and we propose that the strength and success of this approach, focusing on clinical disease, may have led to a relative down-playing of developments around underlying disease processes that inform design of better preventive treatments. The 2001 GOLD document [1], for example, advanced a disease model based on an abnormal airway "inflammatory response of the lungs", with chronic inflammation involving mainly innate inflammatory activation. This inflammation was then said to damage lung tissue, so reinforcing sustained chronic inflammation and itself leading directly to COPD. "Scar tissue" involving the small airways was mentioned, with the implication that this too was due to ongoing inflammatory processes. This initial hypothetical GOLD model set the scene for all subsequent reports, which largely re-iterated this paradigm of inflammatory disease, including for emphysema referred to under the same umbrella. To an extent this proposal may well be correct, but we would suggest the refinement that much of this inflammation is in the airway lumen, this being one aspect of intermediary mediation between smoking and core remodelling disease, rather than the primary airway wall pathology itself.

An inflammation-predominant disease model of COPD has, we believe, had a major influence on respiratory cultural thinking and on its research and therapeutics agenda. However, it is notable that the GOLD model was only explicitly evidenced (with regard to two mechanistic papers [8,9]) in 2001, neither of which really provided what could be regarded as a convincing picture of innate airway wall inflammation. Thus, in 1957, Leopold and Gough [8] observed what they termed "(post)-inflammatory fibrosis" and destruction of small airways in post mortem lungs, the inflammation being inferred from some inflammatory exudate in the lumen of their post mortem tissue samples and the thickened and purely fibrosed airway wall. In addition, SAETTA et al. [9] studied lungs from smokers both with and without COPD, with the major signal in COPD found to be small airway thickening (the only cellular change in the COPD group compared to the other smokers being an increase in $\mathrm{CD}^{+}$lymphocytes). Notably, there was no suggestion of an increase in macrophages or neutrophils in the airway wall tissues.

Our group extensively reviewed this issue in 2017 [10] and concluded that evidence for this inflammatory hypothesis in any broad sense is minimal. We do not intend to review this literature again here, as this review is intended to be an update and follow-up to that previous document. However, EAPEN et al. [11] published what could be regarded as a uniquely comprehensive analysis of airway wall cellular content in the same year and this did not support (and indeed ran counter to) the airway wall inflammation narrative, at least in any conventional sense and especially in the small airways. Furthermore, this was one of the very few such studies that included a normal control population and total cell counts by sublayer in the airway wall, as well as reporting all inflammatory/immune cell types by both absolute number and percentage of total. The most striking feature of airway wall cellularity throughout the bronchial tree in COPD was hypocellularity. This was quite marked, with a $50 \%$ reduction in total cells in smoker COPD in the large airways and $30 \%$ in the small airways (figure 1 , where the larger left-hand chart represents the normal cellular pattern and the smaller right-hand chart the fewer cells of the relatively hypocellular COPD small airway wall). In fact, this replicated an earlier independent analysis, albeit one performed for a different purpose [12]. There are some subtle differences in cells between the large and small airways, and subtle changes in "inflammatory" cells in percentage terms in COPD; however, the only significant change in absolute cell counts was again in $\mathrm{CD}^{+}$lymphocytes. Eosinophils were highly variable between individuals and sites, and much fewer in number than other cell types in what was a typical and non-asthma-hybrid COPD group. Such counts were not obviously different from normal and, as such, eosinophils were not reported due to the poor and uncertain signal. Very importantly, all cellular changes in COPD airways were undoubtedly present as a feature of smoking per se, with only a small extra signal in COPD at most, at least in mild-moderate disease.

One potentially important and rather different report that might unsettle this conclusion on the airway wall, at least regarding COPD itself, was that of PoLosuknin et al. (2017) [13]. Whole lungs were obtained from clinical transplant services for both normal control tissue and ex-smoker severe (stage 3-4) COPD tissue. As in our own small airway studies, for stage 1-2 COPD they obtained lung resection material from ex-smokers with lung cancer. No actively smoking COPD subjects or actively smoking non-COPD subjects were included. Using low power magnification for assessment they found patchy disease that affected some airways and not others, and which was more common in more severe stages of disease 


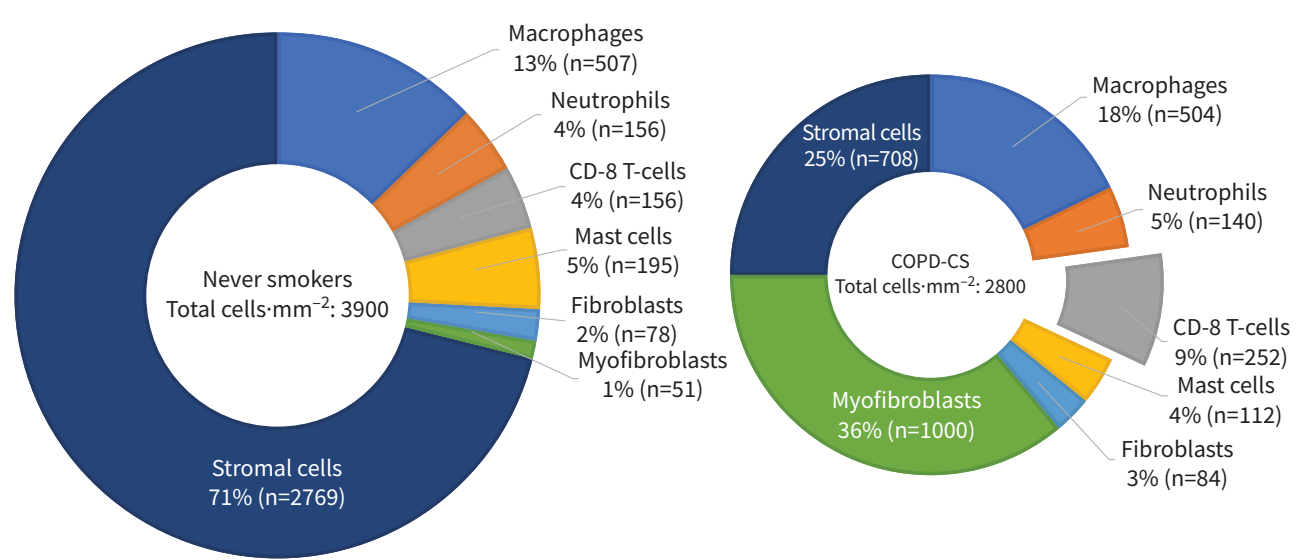

FIGURE 1 Total cell counts and absolute/percentage values for cell types in the small airways of normal never smokers and currently smoking chronic obstructive pulmonary disease (COPD) subjects (COPD-CS subjects). The changes evident in COPD are in fact mainly smoking-related [11, 14]. The size of the pie-charts corresponds to the total cell counts (non-scaled). CS: cigarette smoke.

compared with milder disease. In terms of inflammation, they described neutrophil and macrophage increases that were especially marked in severe disease. These were mostly in the form of peripheral cuffing around the airways in the adventitial layer and the parenchymal lung beyond this, but only in patches where remodelling was also present. Although this may prove to be a highly significant paper, no one else has (to our knowledge) described such marked peripheral inflammatory findings. This applies even to Leopold and Gough [8] in their original detailed descriptions of airway disease in centrilobular emphysema. We would agree with the patchy nature of COPD small airway disease and, when averaged, many remodelling indices have wide group confidence limits and overlap with normal controls in a manner that is consistent with this. Furthermore, human studies of inflammatory cells in the airway wall in COPD have largely been solely about cell profiles. Thus, it is possible that subtle changes in function could be important pathogenically, even with normal cell numbers [11, 14].

Airway remodelling as the primary disease in COPD airways

Airway wall tissue remodelling was first noted early in research into smoking and COPD pathology, with changes to the epithelium (such as goblet cell hyperplasia and squamous metaplasia) [15, 16] accompanied by submucosal (below the muscle layer) mucous gland hyperplasia. However, there is unanimity in the subsequent literature that the most outstanding change in airway resistance in COPD occurs not with these ubiquitous smoking-related epithelial changes but with wall thickening and narrowing, most importantly in the small airways (those of $<2 \mathrm{~mm}$ internal diameter) [17-19]. Despite the physiological resistive consequences being peripheral, all these pathological remodelling changes occur throughout the airway tree and in smokers as well as in COPD subjects. As an aside, the increase in resistance occurs primarily in the middle-sized airways in asthma due to muscle thickening, but becomes peripheral in asthmatic subjects who smoke [20].

In smokers, the first physiological consequences are a decrease in forced vital capacity (FVC) secondary to air trapping and encroachment by increasing residual volume (RV), all due to fixed small airway narrowing [21-23] and air trapping in the surrounding lung. More insights into the crucial role of small airway remodelling in COPD came from the early observations that, as well as wall thickening, the remodelling process involved obliteration of small airways, occurring even before the forced expiratory volume in $1 \mathrm{~s}\left(\mathrm{FEV}_{1}\right) / \mathrm{FVC}$ ratio began to change [19]. This was confirmed later in studies using micro-computed tomography (micro-CT) techniques, indicating that $40 \%$ or more of small airways had disappeared even in apparently earliest-stage COPD [23, 24]. This means that the very ontological designation of COPD, based on a rather arbitrary $\mathrm{FEV}_{1} / \mathrm{FVC}$ ratio $(<70 \%)$, needs updating [5] to avoid underestimating disease burden in smokers.

COPDGene has pointed out how heterogeneous the trajectory to COPD really is [6]. Furthermore, since the conventional transition point is actually quite late in the pathogenic process and given that $\mathrm{FEV}_{1}$ is predominantly a large airway flow index while $\mathrm{FVC}$ itself decreases with air trapping, the $\mathrm{FEV}_{1} / \mathrm{FVC}$ ratio is hardly an optimal measure of deteriorating small airway function. Indeed, in the Australian Burden of 
Obstructive Lung Disease (BOLD) [25] study, which used the degree of scalloping of the mid to distal part of the descending limb of the spirometric flow-volume curve as an appropriate index of small airway narrowing/obliteration, 25\% more significant airway obstruction was detected in the general community.

Thus, one of our main points (now also made by many others [5-7]) is that the focus for prevention and treatment should be more on "smoking-related airway disease" even before progression to COPD, or indeed even before symptoms. Thus, even totally asymptomatic smokers may be in urgent need of secondary prevention measures, especially if they are on a rapid downhill trajectory. While this is frequently inferred it is not in the official guidelines and as such is not yet culturally embedded.

Before moving on, we feel it would be worthwhile to specifically address three pertinent matters. First, the paper perhaps most quoted in defence of the inflammatory model of COPD pathogenesis is probably that from Hogg et al. [26]. Again, the outstanding feature emphasised was airway wall remodelling, but the paper seems more remembered for its small airway wall inflammatory cell profiles in lung resection material. These were from COPD patients only, with no normal or smoker-normal controls. A quite novel technique was used which detected inflammatory-type cells only in a minority of airways in early COPD; thus, only $40 \%$ of all airways contained any neutrophils or macrophages and $20 \%$ had CD8 ${ }^{+}$lymphocytes. This patchiness is again rather reminiscent of the study by PoLOsuKHIN et al. [13], although their cellular findings were very different (in a quantitative cell analysis of the minority of airways containing inflammatory cells, their only significant signal was for $\mathrm{CD}^{+}$lymphocytes and then only in the most severe groups (GOLD 3-4 together)). They further described, again in the most severe COPD only, aggregates of B-cells that were suggested as likely secondary to chronic infection in end-stage disease. Notably, in a rather earlier study [27], our group described such bronchus-associated lymphoid tissue (BALT) aggregates in the airways, with surrounding T-cells and hypervascularity, even in normal subjects (although they were especially common in smokers even without COPD). There has been substantial emphasis given to formulating the pathological picture of COPD based on data from severe disease $[13,26]$. However, it is of interest that the BOLD study emphasised that such end-stage (grade 3-4) disease occurs in only about 2\% of all the COPD in the general population [28]. Thus, as also recently suggested by others [7], we feel that studies on the pathogenesis of COPD would be better focused on early disease and, vitally, on smokers before overt clinical disease appears (where arguably the most important action is to be found).

Secondly, what about bronchoalveolar lavage (BAL)/sputum data? In general, it is accepted that the literature suggests a signal in the airway lumen for innate immune activation, with an increase in macrophages and neutrophils and, in a minority of cases, also in eosinophils (again more in smokers than specifically in COPD cases). Cellular constituents vary quite a lot between BAL and sputum samples, with the former representing peripheral airways and the latter more central airways. Furthermore, at least two independent and thorough reviews of the literature have shown that evidence for the "COPD luminal inflammatory supposition” is rather less certain and more variable than might be generally supposed, even during AEs [10, 29]. One of the issues, especially with sputum, is the presentation of data as percentages of cell-types only [30], without any absolute counts. This makes interpretation difficult because if one cell-type becomes more frequent others must seem fewer, but actually may not have changed at all or may even have increased in absolute number. In addition, one cell-type may seem prominent even when there are actually fewer total cells present. Even so, this approach does seem to have proved useful in discriminating those eosinophil-dominant airways that are responsive to corticosteroid therapy [30] and, in a recent study [30], has proved to be empirically robust enough to show variation in cell percentages related to the molecular microbiome (although this too of course is also semi-quantitative and based on percentage representation of microbe types without reference to quantity).

Thirdly, what about emphysema? GOLD, and especially its 2017 report [4], linked airway and lung parenchymal disease as coincident in pathogenesis and timing. Thus, COPD is "a mix of small airway disease and emphysema due to chronic inflammation" where "inflammation causes (these) structural changes". In fact, there is now very substantial, even clinching, evidence from computer-assisted high-resolution computed tomography (HRCT) lung scanning initially and from voxel-wise parametric response mapping (PRM)-CT scanning subsequently, and also from CT analysis of the morphology of resected lung tissue, that there is very much more small-airway disease present than emphysema (overwhelmingly so in early COPD). Longitudinal studies have confirmed that airway disease precedes parenchymal destruction and indeed that emphysema in smokers occurs where there has been secondary air trapping [24, 31-36].

Epithelial-mesenchymal transition and insights into its role in driving airflow obstruction Epithelial-mesenchymal transition (EMT) is a conserved, fundamental, reversible, biological mechanism underlying embryogenesis/organ development (type 1), wound healing and fibrosis (type 2), and cancer 
development and progression (type 3) [37, 38]. EMT was first postulated as active in human airway disease in 2004 by the Newcastle UK Lung Transplant Group [39-41], as part of the development of bronchiolitis obliterans syndrome (BOS) in chronic rejection [41, 42]. The development of BOS involves airway epithelial injury from a number of causes and, although more sub-acute, resonates with known elements of COPD pathophysiology. This led to a collaborative investigation of the possibility of EMT being active and pathogenic in the airways of smokers with and without COPD. Over the past decade such investigations, from an increasingly large number of centres (including from ourselves) and using increasingly powerful technologies, have largely supported this hypothesis [43]. However, although widely cited in recent reviews on COPD, this work has yet to be fully endorsed in the literature [44, 45].

A seminal paper by CRYSTAL et al. [46] provided evidence that gene reprogramming in airway epithelial basal/stem cells may be a fundamental factor in the development of COPD and this would include well-accepted changes such as squamous cell and goblet cell hyperplasia. We suggest that EMT should be included on that list, which fits with an observation that, as well as being structurally and functionally altered (as described over 60 years ago [15]), there was another prominent feature related to the airway epithelium in smokers and in COPD. Namely that the reticular basement membrane (RBM) immediately below the epithelium's thin true basement membrane (BM) in COPD was not just thickened (as in asthma) but also fragmented, with cell-containing clefts within it, which we realised was a well-accepted classic marker for EMT generally [47]. Confirming the presence of active EMT, we have also shown that both the basal epithelial and RBM cleft cells in smokers stain positively (especially in COPD subjects) for widely used mesenchymal EMT markers such as S100A4, vimentin and $\alpha$-smooth muscle actin ( $\alpha$-SMA), as well as epithelial markers [48].

A series of further clinico-pathologic studies have shown that transforming growth factor- $\beta$ (TGF- $\beta$ ) is likely to have a major EMT-inducing role, working through both its canonical and alternative pathways to activate well-described EMT-specific transcription factors [49, 50], although this is probably along with other growth factor EMT-drivers (table 1 and figure 1). We feel that these findings of reprogrammed airways with active EMT are consistent with and complimentary to the earlier findings of large numbers of destroyed airways even in pre-COPD [31]. Therefore, by definition, our research has been looking at "survivor" airways but has likely been showing the fundamental active pathogenic processes of ongoing small airway wall myofibroblast accumulation, marked wall sub-layer thickening and matrix changes related to luminal narrowing [51]. Ultimately, this would be predicted to lead to luminal obliteration over time, consistent with the important literature from Hogg et al. [31].

A further important novel observation has been that in damaged smoker/COPD airways both the RBM and even the epithelium itself are hypervascular (consistent with type 3 cancer-related EMT), at least in the larger airways. Notably, this feature contrasts with the hypovascularity which accompanies the hypocellularity of the underlying thickened lamina propria [52, 53].

TABLE 1 A summary of selected common stimuli, signalling pathways and transcription factors (TFs) associated with epithelial-mesenchymal transition [37, 38]

\begin{tabular}{|c|c|c|c|}
\hline Selected stimuli & Signalling pathways & TF & TF type \\
\hline TGF- $\beta$ & Smad2, Smad3 and Smad4 ${ }^{\#}$ & Snail1 (Snail) & Zinc finger \\
\hline EGF & MAPK/ERK 1 ฯ & Snail2 (Slug) & Zinc finger \\
\hline VEGF & Wnt $/ \beta$-catenin ${ }^{+}$ & ZEB1 & Zinc finger \\
\hline HGF & Notch $^{\S}$ & ZEB2 (SIP1) & Zinc finger \\
\hline Bile acids & Hedgehog $^{f}$ & Twist1 & Basic helix-loop helix \\
\hline
\end{tabular}

The Snail, Twist and ZEB TF families control cell-cell adhesion, cell migration and extracellular matrix (ECM) degradation. They have been shown to have evolutionarily conserved roles in epithelial-mesenchymal transition (EMT) in different biological settings and in a range of organisms. TGF- $\beta$ : transforming growth factor- $\beta$; EGF: epidermal growth factor; VEGF: vascular endothelial growth factor; HGF: hepatocyte growth factor; MAPK: mitogen-activated protein kinase; ERK: extracellular signal-regulated kinase; ZEB: zinc finger E-box-binding homeobox; SIP: Smad interacting protein. \#: TGF- $\beta$ (the classic, positive control in in vitro EMT experiments) activates Smad2 and Smad3 and complexes with Smad4, which then interacts with DNA-binding TFs inducing EMT; an alternative pathway operates via $\beta$-catenin released when intercellular adhesion breaks down; I: activated by EGF-receptor stimulation for example; ${ }^{+}$: the Wnt pathway causes cytoplasmic $\beta$-catenin accumulation and nuclear translocation to act as a TF co-activator; ${ }^{\S}$ : Notch signalling activation occurs via cell to cell contact with ligand expression in signal-providing cells (dysregulated in the epithelium of COPD patients); ${ }^{f}$ : EMT/lung cancer are linked to the Hedgehog signalling pathway, which is modulated by cigarette smoke. 
Other groups have also demonstrated EMT in COPD airways and there have been $>100$ papers in PubMed on this subject in the last decade, mainly in the last 5 years. To quote just one of these, GoHy et al. [54] used complimentary primary airway epithelial cultures and airway biopsies from COPD patients to show that the bronchial epithelium from the conducting airways of COPD patients exhibited increased mesenchymal vimentin expression with decreased epithelial ZO-1 and E-cadherin protein expression, consistent with EMT in vivo. The increase in vimentin expression correlated with BM thickening and airflow limitation. Importantly, the mesenchymal features of the COPD epithelial cells in vivo were "recapitulated" in ex vivo air-liquid interface cultures. The air-liquid interface process involves cell expansion in culture and passage, so this demonstrates a strong imprinting of the COPD airway epithelial gene fingerprint despite being removed from injury and the airway milieu.

Chronic smoking seems to interact with a range of other airway damaging factors in activation of epithelial EMT. The ECLIPSE study [55] found that the second-best predictor of COPD exacerbations was a patient history of gastroesophageal reflux, likely linked to micro-aspiration. Of relevance to this, CHEN et al. [56] showed that coincident expression of (bile acid) farnesoid X receptors (FXR) and EMT markers was significantly elevated in the small airway epithelium of COPD patients and was related to airflow obstruction. In a similar vein of extraneous interactions with a highly relevant COPD comorbidity, a recent paper from $\mathrm{Wu}$ et al. [57] indicated that high glucose tensions combined with cigarette smoke (CS) challenge induced EMT in a rat model of diabetes and in cultured human airway cells, and that patients with COPD and diabetes had worse airway obstruction than COPD patients without diabetes.

Although the details are beyond the scope of this article, associations of COPD with lung cancer specifically (even allowing for smoking) are well accepted and of high clinical and epidemiological significance. This association has also increasingly been linked with EMT and its known drivers such as TGF- $\beta 1$ (table 1) [58-61]. Chronic fibrotic processes are generally strongly associated with epithelial cancer formation, again with TGF- $\beta 1$-induced EMT intimately implicated in that association (the so-called "wound healing, chronic fibrosis and cancer progression triad”) [62, 63]. A novel clinical pathology study by MAнмоор et al. [64] has directly related prognostic EMT activity in nonsmall cell lung cancers with that in the same patients' non-cancerous airways.

Of human cancers, 95\% are of epithelial origin and EMT is increasingly regarded as being inherent to epithelial cancer development in general, as well as metastasis, poor prognosis and drug resistance [64]. Fortunately, promising anti-EMT drugs are now being developed to inhibit these processes in a wide number of specific tumours, with the promise of better clinical outcomes [65-73]. As such, novel therapy for EMT in COPD is also potentially feasible.

How do studies on the genetics of COPD and on airway ageing add to this picture?

A number of somatic genes have been implicated in COPD, although mainly as a relatively small contribution to emphysema with (small) airway disease per se being very much less studied, mainly because of the difficulty in specifically phenotyping it radiologically or physiologically [74]. The only gene strongly associated with the non-emphysema phenotype of COPD (i.e. airway disease) is FAM13A on chromosome 4q22 [75], which seems especially related to instability of cell-cell adhesion resulting in release of $\beta$-catenin into the cell cytoplasm, a well-known, strong stimulus to EMT and tissue fibrosis.

In a systems biology approach, WANG et al. [76] recently described mutual gene expression profiles and genetic interaction networks for the bronchial epithelium and lung cancer. One standout active gene was $T B L 1 X R 1$, which is involved in the epigenetic interaction between histones and EMT-like activity, and myofibroblast proliferation, with high activity relating to poor squamous lung cancer survival as well as to risk of COPD. Paradoxically, in terms of current medications for asthma/COPD, a risk factor for both COPD and lung cancer is upregulation of the ADRB2 gene for sympathetic $\beta 2$ receptors, with downregulation of this gene activity associated with better squamous-cell cancer survival [76]. Using 3D epithelial cell culture, Matsumura et al. [77] exposed cells to oxidant stress and found 15 genes were perturbed, with those for cell proliferation and redox homeostasis being downregulated; however, EMT-related genes (including for epidermal growth factor receptor (EGFR)) were upregulated and this was especially marked in cells from smokers and COPD patients.

In an analysis of epigenetic methylated gene profiles as an index of gene upregulation, KACHROO et al. [78] found that in COPD tissue there was a prominence of gene methylation associated with the TGF- $\beta$, Wnt, Notch and Hedgehog EMT-signalling pathways, including phosphoinositide 3-kinase (PI3K)-protein kinase B (Akt), a kinase closely related to EMT activation [79]. 
In their recent review of genetics in COPD, SILVERMAN et al. [74] emphasised increasing current interest in COPD and single nucleotide polymorphism (SNP)-based allelic variants. As an example, Zнао et al. [80] showed a strong COPD risk associated with the C1562T allelic variant of the matrix metallopeptidase (MMP)-9 gene. MMP-9 expression in the epithelium is a strong signal for EMT activity, as it is involved in digesting a pathway though the RBM for motile mesenchymally transitioning epithelial cells. Similarly, Devries and Vercelli [81] provided strong evidence for involvement of variants in TGF- $\beta$ pathway genes in COPD. Indeed, particularly disadvantageous alleles of (anti-oxidant enzyme) glutathione S-transferase (GST) genes favour loss of airflow with even low amounts of oxidising pollution [82, 83].

The concept that COPD may represent an acceleration of a genetically determined normal lung ageing process has gained traction following a mainly hypothesis-generating paper by SANDFords et al. [84]. This showed that cultured epithelial cells stressed by CS extract produced a senescence marker, $\beta$-galactosidase and that alveolar type 2 cells from emphysematous lung tissue demonstrated tell-tale shortened telomeres, as did blood leukocytes from the same individuals. Later, shorter telomere length was related to increased morbidity and mortality in COPD [85]. However, the picture has become far from clear and indeed quite complex, with studies of polymorphisms of classic ageing genes (namely TERT, DSP and FAM13A) showing a picture typical of senescence with reduced expression in idiopathic pulmonary fibrosis (IPF) lungs, but paradoxically anti-senescent high expression in both COPD and lung cancer [86]. There was however a muted and partial pattern of senescence observed rarely in emphysema. These "inverse-senescence" genetic traits in (airway) COPD/cancer would slow cell turnover, decrease apoptosis and increase cell longevity. All of these functional cell changes would allow extra time for accrued DNA damage to present as malignant transformation and would also have a pro-EMT/airway fibrosis effect [86].

In a recent review, OcchipINTI et al. [87] catalogued the known consensus radiological features of the ageing lung: large airway wall thinning, large and medium sized airway luminal dilatation, air trapping, decreased cillial function, increased luminal secretion, alveolar dilatation with decreased surface area, reduced lung vessels (with preserved ventilation $(V)$ /perfusion $(Q)$ matching) and finally an increase in linear and reticular shadowing. Emphysema (i.e. alveolar destruction) was not feature and nor was airway wall thickening. Indeed, airways were thought to be abnormally compliant, as were lung parenchyma [87]. One study reviewed in this latter article reported data on 4th to 6th generation airways using computer-assisted HRCT lung scanning [88] in healthy, non-smoking subjects aged 60-90 years, with air trapping being the most prominent feature. This would most likely be due to small airway dysfunction but there was no evidence of distal airway thickening. There was also dilatation of mid-sized airways. A later HRCT study [89] provided quantitative information on airways down to $3.5 \mathrm{~mm}$ external diameter in healthy individuals, but only up to an age of 55 years. It found progressive full-length airway wall thinning with age, although in contrast it found generalised airway thickening in smokers.

Finally, using a novel method of endobronchial optical coherence tomography, Su et al. [90] showed that the morphology of the normal airway did change with age between 20 and 69 years of age, with thinning of cartilage and dilatation of the airway in middle age (with secondary narrowing after the age of 55 years), but with no apparent change in airway wall thickening.

Taking all this information together, it seems most likely that although there may be some overlap, COPD is not just accelerated ageing but a distinct and quite specific pathological process, one that is linked to lung cancer. One feature of COPD that does definitely overlap with airway ageing is air trapping; however, in normal ageing this is likely to be due to decreased lung elastic recoil and also perhaps greater compliance of the airways themselves. By contrast, the main and certainly early physiological problem in COPD is small airway thickening, luminal narrowing and destruction. Thus, COPD seems most likely to be a disease "in" the ageing process, rather than "of" it per se. Indeed, COPD pathology seems to shift the natural senescence history of the lung largely in a different yet disastrous direction.

Infection as a driver of airway luminal inflammation and epithelial activation

Recurrent microbial infection and/or colonisation of the airway with bacteria, viruses or both together remains a crucial symptom-generating part of COPD and especially AEs. Indeed, infections are linked to approximately $85 \%$ of AEs [55]. However, aside from symptoms and exacerbations, chronic airway luminal infection/colonisation in and of itself results in increased chronic morbidity and mortality [91].

As documented extensively, the most frequent micro-organisms isolated from the lower respiratory tract of even asymptomatic smokers and of persistently colonised COPD patients are non-typeable Haemophilus influenzae (NTHi), Streptococcus pneumoniae and Pseudomonas aeruginosa (though the latter is mainly seen in the more severe stages) [91, 92]. Furthermore, NTHi is also the bacterial pathogen most frequently 
isolated from patients with AEs of COPD [93, 94]. Colonisation of the lower respiratory tract by these “classic/typical” respiratory bacterial pathogens is also associated with an increase in daily symptoms and increased sputum interleukin (IL)-8 levels [95], as well as very importantly being a driver of accelerated decline in $\mathrm{FEV}_{1}$ [96].

A number of possible mechanisms underlying this major airway bacterial infection problem in COPD have been suggested, mainly a hypothesised "immune defect" (such as a focal decrease in epithelial surface secretory IgA) [13] or smoking-induced high oxidative stress (and/or epithelial barrier dysfunction) [97]. We have highlighted another pathogenic pathway, the absolute need for microbes to adhere to serendipitous receptors on the airway epithelium before they can colonise/infect/invade. We have found that the known major binding receptor for all these classic respiratory bacteria to adhere, platelet-activating factor (PAF) receptor, is significantly upregulated in expression in the epithelium of both the large and small airway epithelia in smokers and even more so in those with COPD. Moreover, PAF receptor expression is strongly associated with the scale of smoking history [98, 99]. Furthermore, none of these mechanisms need be mutually exclusive.

In the airways, PAF itself may modulate host inflammation [100-102]; however, PAF receptor upregulation seems to be utilised coincidentally as a convenient and abundant adhesion site in smokers for all the major respiratory bacterial pathogens highlighted above [103]. Furthermore, blocking PAF receptor with specific receptor antagonists counters the epithelial adhesion of $H$. influenzae, S. pneumoniae and $P$. aeruginosa in cultured lung epithelial cells $[104,105]$. The adhesion of these bacteria to PAF receptor is due to them all having, quite unusually and serendipitously, the molecule phosphorylcholine (ChoP) as a binding site precisely positioned enzymatically in their outer lipopolysaccharide layer [106]. In addition, variation in ChoP genes through epigenetic control relates to bacterial pathogenicity [107, 108].

These "microbial adhesion” insights have led us to a general proposal for further clinical assessment of non-antibiotic approaches to the inhibition of microbial pathology, for both bacteria and viruses, by inhibiting their adhesion to the respiratory mucosa [109]. Drugs already available, such as the PAF receptor blockers trialled as anti-asthma therapies over 25 years ago, could potentially be useful here and could be used as prevention/prophylaxis treatments or in the initial phases of AEs.

Finally, persistence of low-grade airway luminal inflammation secondary to chronic infection and its generated reactive oxygen species (ROS), or the microbes themselves, may well drive epithelial activation, gene reprogramming and TGF- $\beta 1$, tumour necrosis factor- $\alpha$ (TNF- $\alpha$ ) and Wnt production, which then drive EMT activation [109-111] (figure 2). This suggests an integrated model of such drivers in the lumen that is intimately involved with what happens to the epithelium and through it to the tissues below where the directly obstructive pathology occurs.

\section{Potential role of oxidant stress}

The oxidation of critical and potentially damaging cellular molecules in smokers' airways by ROS and other acute phase oxidants is, as in other tissues, dealt with through the antioxidant glutathione and the multifaceted GST enzyme system. This is responsible for the S-glutathionylation of potentially damaging oxidised reactive electrophilic centres on a wide variety of substrates, such as thiol groups on protein/ enzyme cysteine residues and lipid peroxidation sites, in order to make the compounds more water soluble to aid detoxification and elimination from the cell. GSTs also have an important separate role in control of the complex kinase cellular systems involved in vital functions such as cell proliferation, apoptosis and EMT [112]. Indeed, this system seems to be a vital link between ROS-induced oxidant stress on epithelial basal cells, TGF- $\beta$ production, induction of EMT with fibrosis and also malignancy. The GST system is negatively controlled by another enzyme, glutaredoxin and its deficiency in epithelial cells has been shown to increase activation of the TGF- $\beta$ pathway and increase airway fibrotic remodelling [112]. GST gene polymorphisms have also been mentioned as favouring loss of airflow [82, 83].

\section{Conclusions: relevance of this model to therapeutics in COPD}

As we have shown, it is hardly a novel proposition that the key underlying features of COPD (and also of smokers in pre-COPD) are airway wall tissue remodelling and thickening, and ultimately loss of a large number of small airways. However, we do appreciate that pathologies in the airway lumen induced by CS, including inflammation and oxidant stress, may well be important as intermediary determinants of epithelial activation to set this process off. Overall, we would encourage future guideline statements to reappraise the totality of evidence on pathology and ponder how this integrated whole could change the focus of the suggested management strategies, especially if we can nominate relevant pathogenic 


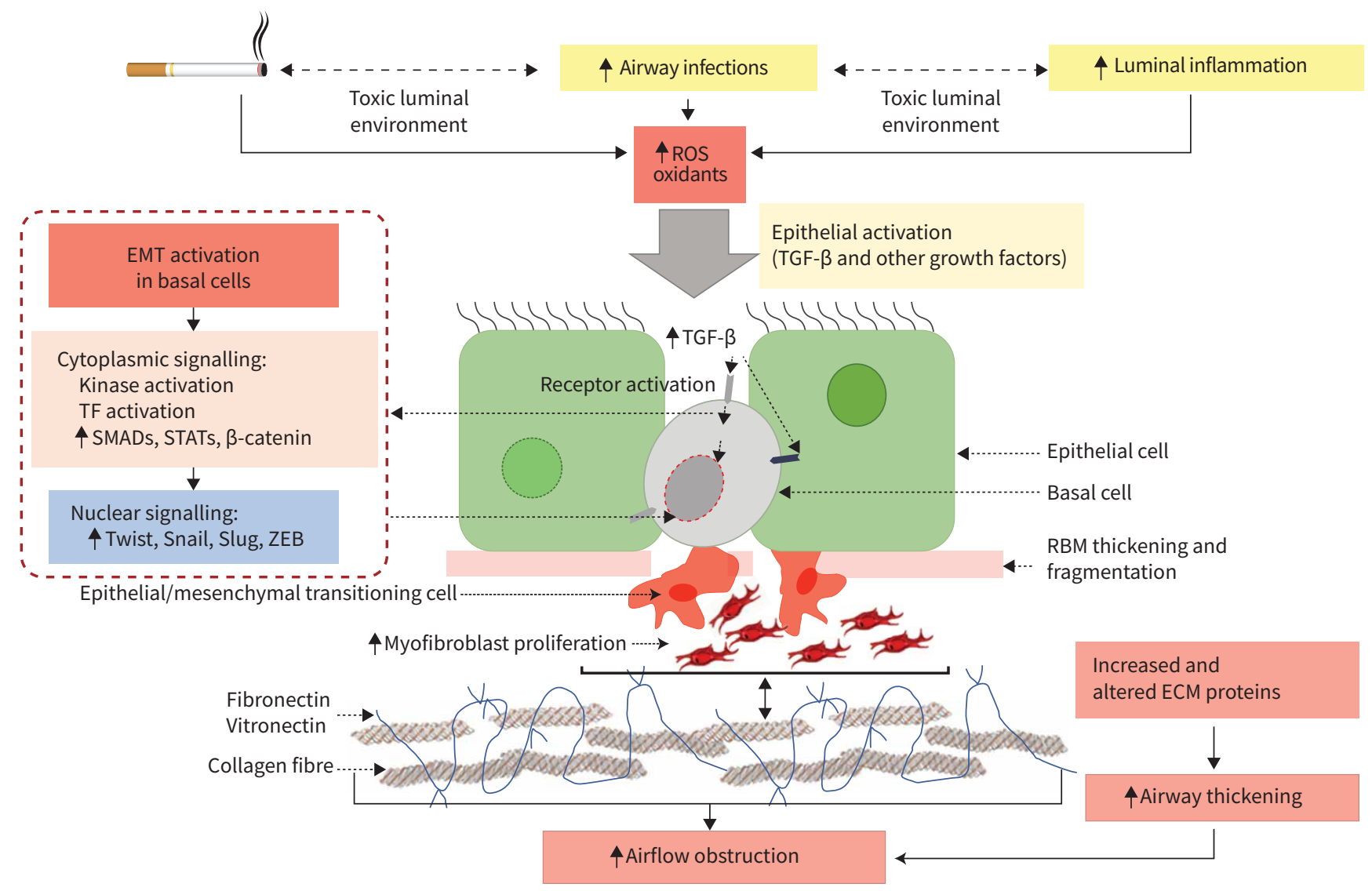

FIGURE 2 Schematic of epithelial-mesenchymal transition (EMT) in cigarette smoke (CS)-related chronic obstructive pulmonary disease (COPD). Chronic CS exposure leads to alteration of the airway luminal environment (e.g. oxidants, infection and inflammation). The increase in reactive oxygen species (ROS) activates the respiratory epithelium, with an increase in transforming growth factor- $\beta$ (TGF- $\beta$ ) as well as other EMT-driver production. In turn, this activates basal cell cytoplasmic signalling (via Smads, STATs, $\beta$-catenin and kinase reactions), to produce further nuclear transcription factors (such as Twist, Snail, Slug and ZEB). All this activity leads to the initiation of transition of basal cells into mesenchymal cells, rather than the normal/abnormal epithelial phenotype. This results in proliferation of myofibroblasts which then markedly increase secretion of both standard and abnormal extracellular matrix (ECM) proteins, leading to airway thickening/obliteration and subsequent airflow limitation. TF: transcription factor; RBM: reticular basement membrane; STAT: signal transducer and activator of transcription; ZEB: zinc finger E-box-binding homeobox.

pro-fibrotic processes as targets. An overview model of what we think are such a broad set of COPD-related processes is given in figure 3.

We have, in particular, shown that EMT plays a major (and possibly fundamental) final pathway role in COPD (small) airway remodelling disease. EMT is now increasingly being considered as treatable, at least in oncology [65-73], while in IPF anti-fibrotic drugs (such as nintedanib and pirfenidone) [113] have been introduced with effect. All of these drug modalities hold great promise for translation to COPD; however, drugs already used in COPD may themselves have an anti-EMT effect, either directly and/or through damping innate luminal inflammation and related secondary ROS attack. It is notable that inhaled corticosteroids (ICS) have been suggested as being both protective in lung cancer [114] and specifically inhibitory of EMT in human airways [115]. Long-acting $\beta$-agonists (LABAs), long-acting muscarinic antagonists (LAMAs) and phosphodiesterase (PDE)-inhibitors may also contribute to a direct anti-EMT effect [113, 116], as might urokinase-type plasminogen activator receptor (uPAR) inhibition [117]. In the same vein, statins have long been thought of as potentially useful in COPD, both in terms of morbidity and mortality, but have been shown not to help in regard to frequency of exacerbations [118]. They also decrease lung cancer risk [119] while being shown to reduce EMT activity and through this cancer aggressiveness [120]. Designer small molecules which inhibit the epigenetic reader BRD4 have been shown to switch off EMT activity and airway remodelling via a TGF- $\beta$ related mechanism [121]. It may well be that our current focus on symptoms and short-term outcomes, to the detriment of long-term 


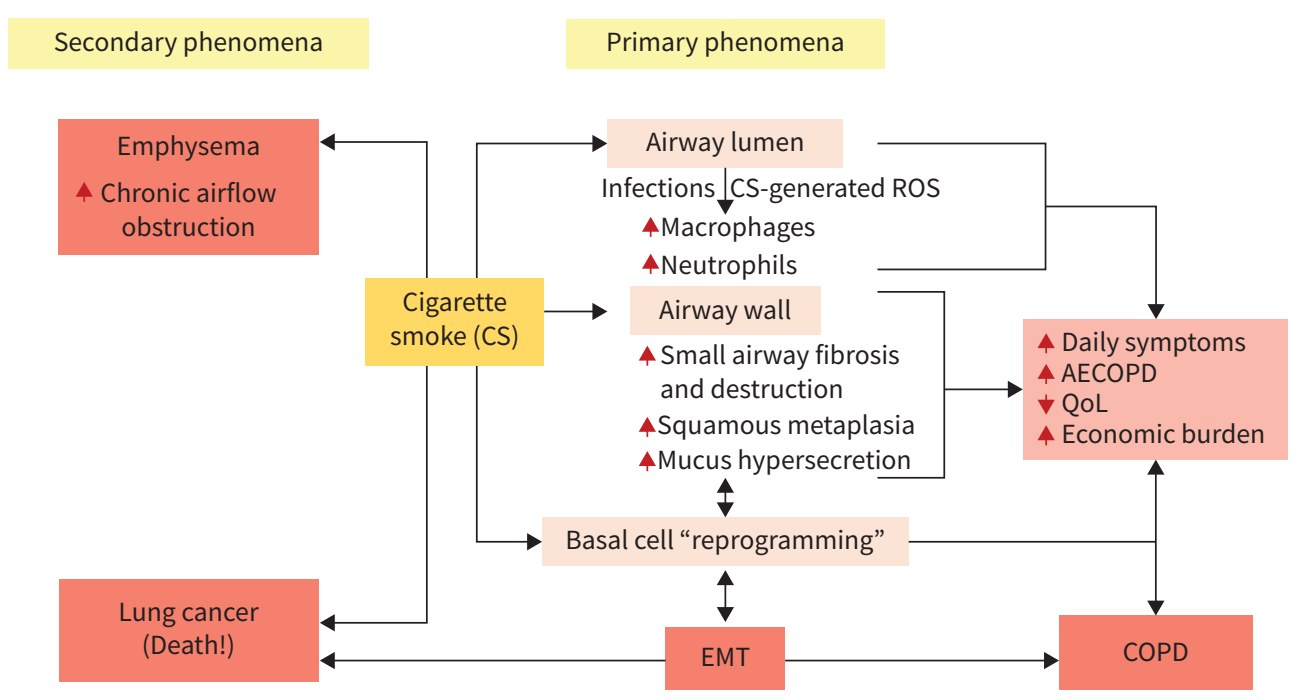

FIGURE 3 Overall pathogenesis of chronic obstructive pulmonary disease (COPD). Primary phenomena include airway luminal cellular inflammation (due to chronic infection with "respiratory pathogens" and/or cigarette smoke (CS)-induced oxidative burden) and fibrosis and obliteration of the small airways, as well as remodelling changes including squamous metaplasia and mucus hypersecretion, and goblet-cell hyperplasia in the large airways. These changes together are ultimately associated with daily symptoms, risk of acute exacerbations of COPD (AECOPDs), economic burden and decreased quality of life (QoL). Basal cells in the airway epithelium are increasingly regarded as extremely important in airway remodelling in all its forms, including those resulting from epithelial-mesenchymal transition (EMT) and angioneogenesis. Secondary phenomena include emphysema (enlargement and destruction of terminal bronchioles and alveoli) and lung cancer, likely related to EMT.

remodelling (as well as cancerogenic and functional outcomes in COPD), has led our research community to overlook the potential of repurposing common drugs, while maybe not utilising to its full potential its ability to trial novel drugs already in the pipeline.

We propose that with such EMT-related therapies being available, either now or in the near future, there are exciting opportunities to trial them within the emerging framework of the call for COPD management to be reviewed. We need to become more focused on detecting and managing core pathologies in at-risk individuals as early as possible and in preventing progression to clinical symptomatic disease [5-7]. Recent long-term cohort and epidemiological studies have shown that this is feasible and necessary, and they have helped us to better understand the natural history and phenotypes of both pre-COPD and established COPD [6, 7, 121, 122]. Such an approach needs a concerted push into primary care to obtain individualised spirometry baselines in early middle age and then within subsequent trajectories. Such baselines need acceptance as necessary routine screening and prevention tools, akin to those for blood pressure, cholesterol and HbA1C levels.

Within the profession we perceive an urgent need to build on the great progress made by GOLD and other initiatives, and a need to better understand and integrate the various pathologies that affect the interconnected airway lumen, epithelium and sub-epithelium (without which we cannot move forward) [7]. COPD is undoubtedly a complex disease with a number of dimensions and we need to know how all of these fit together in order to develop new conceptions of what can be achieved for our patients.

Provenance: Submitted article, peer reviewed.

Author contributions: E.H. Walters conceptualised the manuscript. All authors contributed to the literature search, as well as the writing and revision of the manuscript. S.D. Shukla and E.H. Walters designed the figures.

Conflict of interest: None declared.

Support statement: E.H. Walters was supported for the work described by a Senior Practitioner Fellowship and Centre of Research Excellence program award from the NHMRC (Australia). S.D. Shukla is supported by research 
funding from the School of Medicine and Public Health, Faculty of Health and Medicine and Priority Research Centre for Healthy Lungs, University of Newcastle, NSW, Australia. C. Ward is supported by the Medical Research Foundation and an unrestricted grant from Boehringer Ingelheim.

\section{References}

1 Pauwels RA, Buist AS, Calverley PM, et al. Global strategy for the diagnosis, management, and prevention of chronic obstructive pulmonary disease. NHLBI/WHO Global Initiative for Chronic Obstructive Lung Disease (GOLD) Workshop summary. Am J Respir Crit Care Med 2001; 163: 1256-1276.

2 Rabe KF, Hurd S, Anzueto A, et al. Global strategy for the diagnosis, management, and prevention of chronic obstructive pulmonary disease: GOLD executive summary. Am J Respir Crit Care Med 2007; 176: 532-555. Vestbo J, Hurd SS, Agustí AG, et al. Global strategy for the diagnosis, management, and prevention of chronic obstructive pulmonary disease: GOLD executive summary. Am J Respir Crit Care Med 2013; 187: 347-365.

4 Vogelmeier CF, Criner GJ, Martinez FJ, et al. Global strategy for the diagnosis, management, and prevention of chronic obstructive lung disease 2017 report. GOLD executive summary. Am J Respir Crit Care Med 2017; 195: 557-582.

5 Agusti A, Alcazar B, Cosio B, et al. Time for a change: anticipating the diagnosis and treatment of COPD. Eur Respir J 2020; 56: 2002104.

6 Lowe KE, Regan EA, Anzueto A, et al. COPDGene ${ }^{\circledR}$ 2019: redefining the diagnosis of chronic obstructive pulmonary disease. Chronic Obstr Pulm Dis 2019; 6: 384-399.

7 Martinez FJ, Han MK, Allinson JP, et al. At the root: defining and halting progression of early chronic obstructive pulmonary disease. Am J Respir Crit Care Med 2018; 197: 1540-1551.

8 Leopold JG, Gough J. The centrilobular form of hypertrophic emphysema and its relation to chronic bronchitis. Thorax 1957; 12: 219-235.

9 Saetta M, Di Stefano A, Turato G, et al. CD8 ${ }^{+}$T-lymphocytes in peripheral airways of smokers with chronic obstructive pulmonary disease. Am J Respir Crit Care Med 1998; 157: 822-826.

10 Eapen MS, Myers S, Walters EH, et al. Airway inflammation in chronic obstructive pulmonary disease (COPD): a true paradox. Expert Rev Respir Med 2017; 11: 827-839.

11 Eapen MS, McAlinden K, Tan D, et al. Profiling cellular and inflammatory changes in the airway wall of mild to moderate COPD. Respirology 2017; 22: 1125-1132.

12 Sohal SS, Reid D, Soltani A, et al. Changes in airway histone deacetylase2 in smokers and COPD with inhaled corticosteroids: a randomised controlled trial. PLoS One 2013; 8: e64833.

13 Polosukhin VV, Richmond BW, Du RH, et al. Secretory IgA deficiency in individual small airways is associated with persistent inflammation and remodeling. Am J Respir Crit Care Med 2017; 195: 1010-1021.

14 Eapen MS, Hansbro PM, McAlinden K, et al. Abnormal M1/M2 macrophage phenotype profiles in the small airway wall and lumen in smokers and chronic obstructive pulmonary disease (COPD). Sci Rep 2017; 7: 13392.

15 Reid L. Measurement of the bronchial mucous gland layer: a diagnostic yardstick in chronic bronchitis. Thorax 1960; 15: 132-141.

16 Thurlbeck WM, Angus GE. A distribution curve for chronic bronchitis. Thorax 1964; 19: 436-442.

17 Dunnill MS. The classification and quantification of emphysema. Proc R Soc Med 1969; 62: 1024-1027.

18 Macklem PT. The physiology of small airways. Am J Respir Crit Care Med 1998; 157: S181-S183.

19 Macklem PT. A century of the mechanics of breathing. Am J Respir Crit Care Med 2004; 170: 10-15.

20 Despas PJ, Leroux M, Macklem PT. Site of airway obstruction in asthma as determined by measuring maximal expiratory flow breathing air and a helium-oxygen mixture. J Clin Invest 1972; 51: 3235-3243.

21 Hogg JC, Macklem PT, Thurlbeck WM. Site and nature of airway obstruction in chronic obstructive lung disease. N Engl J Med 1968; 278: 1355-1360.

22 Peter T, Macklem WM, Thurlbeck RGF. Chronic obstructive disease of small airways. Ann Intern Med 1971; 74: 167-177.

23 Cosio M, Ghezzo H, Hogg JC, et al. The relations between structural changes in small airways and pulmonary-function tests. N Engl J Med 1978; 298: 1277-1281.

24 McDonough JE, Yuan R, Suzuki M, et al. Small-airway obstruction and emphysema in chronic obstructive pulmonary disease. N Eng J Med 2011; 365: 1567-1575.

25 Johns DP, Walters JA, Walters EH. Diagnosis and early detection of COPD using spirometry. $J$ Thorac Dis 2014; 6: 1557-1569.

26 Hogg JC, Chu F, Utokaparch S, et al. The nature of small-airway obstruction in chronic obstructive pulmonary disease. N Engl J Med 2004; 350: 2645-2653.

27 Richmond I, Pritchard GE, Ashcroft T, et al. Bronchus associated lymphoid tissue (BALT) in human lung: its distribution in smokers and non-smokers. Thorax 1993; 48: 1130-1134.

28 Toelle BG, Xuan W, Bird TE, et al. Respiratory symptoms and illness in older Australians: the Burden of Obstructive Lung Disease (BOLD) study. Med J Aust 2013; 198: 144-148. 
Wang Y, Xu J, Meng Y, et al. Role of inflammatory cells in airway remodeling in COPD. Int J Chron Obstruct Pulmon Dis 2018; 13: 3341-3348.

Wang Z, Locantore N, Haldar K, et al. Inflammatory endotype associated airway microbiome in COPD clinical stability and exacerbations - a multi-cohort longitudinal analysis. Am J Respir Crit Care Med 2020; in press [https://doi.org/10.1164/rccm.202009-34480C].

Hogg JC, Paré PD, Hackett TL. The contribution of small airway obstruction to the pathogenesis of chronic obstructive pulmonary disease. Physiol Rev 2017; 97: 529-552.

Boes JL, Hoff BA, Bule M, et al. Parametric response mapping monitors temporal changes on lung CT scans in the subpopulations and intermediate outcome measures in COPD study (SPIROMICS). Acad Radiol 2015; 22: 186-194.

Bhatt SP, Soler X, Wang X, et al. Association between functional small airway disease and $\mathrm{FEV}_{1}$ decline in chronic obstructive pulmonary disease. Am J Respir Crit Care Med 2016; 194: 178-184.

Koo HK, Vasilescu DM, Booth S, et al. Small airways disease in mild and moderate chronic obstructive pulmonary disease: a cross-sectional study. Lancet Respir Med 2018; 6: 591-602.

Labaki WW, Gu T, Murray S, et al. Voxel-wise longitudinal parametric response mapping analysis of chest computed tomography in smokers. Acad Radiol 2019; 26: 217-223.

Colak Y, Afzal S, Nordestgaard BG, et al. Prevalence, characteristics, and prognosis of early chronic obstructive pulmonary disease. The Copenhagen general population study. Am J Respir Crit Care Med 2020; 201: 671-680.

Kalluri R. EMT: when epithelial cells decide to become mesenchymal-like cells. J Clin Invest 2009; 119: 1417-1419.

Yang J, Antin P, Berx G, et al. Guidelines and definitions for research on epithelial-mesenchymal transition. Nat Rev Mol Cell Biol 2020; 21: 341-352.

Ward C, Forrest IA, Lordan JL, et al. Epithelial to mesenchymal transition in clinically stable lung transplantation. Transplantation 2004; 78: 190. https://journals.Iww.com/transplantjournal/Fulltext/2004/07271/ Epithelial_to_Mesenchymal_Transition_in_Clinically.496.aspx.

Ward C, Robertson H, Forrest IA, et al. Hypothesis: epithelial-to-mesenchymal transition is a common cause of chronic allograft failure. Transplant Proc 2005; 37: 977-980.

Ward C, Forrest IA, Murphy DM, et al. Phenotype of airway epithelial cells suggests epithelial to mesenchymal cell transition in clinically stable lung transplant recipients. Thorax 2005; 60: 865-871.

Borthwick LA, Parker SM, Brougham KA, et al. Epithelial to mesenchymal transition (EMT) and airway remodelling after human lung transplantation. Thorax 2009; 64: 770-777.

Eapen MS, Sharma P, Gaikwad AV, et al. Epithelial-mesenchymal transition is driven by transcriptional and post transcriptional modulations in COPD: implications for disease progression and new therapeutics. Int $J$ Chron Obstruct Pulmon Dis 2019; 14: 1603-1610.

Bartis D, Mise N, Mahida RY, et al. Epithelial-mesenchymal transition in lung development and disease: does it exist and is it important? Thorax 2014; 69: 760-765.

Higham A, Quinn AM, Cançado JED, et al. The pathology of small airways disease in COPD: historical aspects and future directions. Respir Res 2019; 20: 49.

Shaykhiev R, Crystal RG. Basal cell origins of smoking-induced airway epithelial disorders. Cell Cycle 2014; 13: 341-342.

Sohal SS, Reid D, Soltani A, et al. Reticular basement membrane fragmentation and potential epithelial mesenchymal transition is exaggerated in the airways of smokers with chronic obstructive pulmonary disease. Respirology 2010; 15: 930-938.

Sohal SS, Reid D, Soltani A, et al. Evaluation of epithelial mesenchymal transition in patients with chronic obstructive pulmonary disease. Respir Res 2011; 12: 130.

Mahmood MQ, Walters EH, Shukla SD, et al. $\beta$-catenin, Twist and Snail: transcriptional regulation of EMT in smokers and COPD, and relation to airflow obstruction. Sci Rep 2017; 7: 10832.

Mahmood MQ, Reid D, Ward C, et al. Transforming growth factor (TGF) $\beta_{1}$ and Smad signalling pathways: a likely key to EMT-associated COPD pathogenesis. Respirology 2017; 22: 133-140.

Eapen M, Walters $\mathrm{H}$, Hackett T, et al. Small airway fibrosis associated with decreased lung function in COPD (TO 050). Respirology 2018; 23: Suppl. 1, 21-103.

Soltani A, Sohal SS, Reid D, et al. Vessel-associated transforming growth factor-beta1 (TGF- $\beta 1$ ) is increased in the bronchial reticular basement membrane in COPD and normal smokers. PLoS One 2012; 7: e39736.

Soltani A, Walters EH, Reid DW, et al. Inhaled corticosteroid normalises some but not all airway vascular remodeling in COPD. Int J Chron Obstruct Pulmon Dis 2016; 11: 2359-2367.

Gohy ST, Hupin C, Fregimilicka C, et al. Imprinting of the COPD airway epithelium for dedifferentiation and mesenchymal transition. Eur Respir J 2015; 45: 1258-1272.

Hurst JR, Vestbo J, Anzueto A, et al. Susceptibility to exacerbation in chronic obstructive pulmonary disease.

N Engl J Med 2010; 363: 1128-1138. 
Chen B, You WJ, Xue S, et al. Overexpression of farnesoid X receptor in small airways contributes to epithelial to mesenchymal transition and COX-2 expression in chronic obstructive pulmonary disease. $J$ Thorac Dis 2016; 8: 3063-3074.

Wu N, Wu Z, Sun J, et al. Small airway remodeling in diabetic and smoking chronic obstructive pulmonary disease patients. Aging (Albany NY) 2020; 12: 7927-7944.

Wellner U, Schubert J, Burk UC, et al. The EMT-activator ZEB1 promotes tumorigenicity by repressing stemness-inhibiting microRNAs. Nat Cell Biol 2009; 11: 1487-1495.

Bhowmick NA, Zent R, Ghiassi M, et al. Integrin $\beta_{1}$ signaling is necessary for transforming growth factor- $\beta$ activation of p38MAPK and epithelial plasticity. J Biol Chem 2001; 276: 46707-46713.

Gregory PA, Bracken CP, Smith E, et al. An autocrine TGF- $\beta / Z E B / m i R-200$ signaling network regulates establishment and maintenance of epithelial-mesenchymal transition. Mol Biol Cell 2011; 22: 1686-1698.

Yuan Z, Goetz JA, Singh S, et al. Frequent requirement of hedgehog signaling in non-small cell lung carcinoma. Oncogene 2007; 26: 1046-1055.

Mahmood MQ, Sohal SS, Shukla SD, et al. Epithelial mesenchymal transition in smokers: large versus small airways and relation to airflow obstruction. Int J Chron Obstruct Pulmon Dis 2015; 10: 1515-1524.

Rybinski B, Franco-Barraza J, Cukierman E. The wound healing, chronic fibrosis, and cancer progression triad. Physiol Genomics 2014; 46: 223-244.

Mahmood MQ, Ward C, Muller HK, et al. Epithelial mesenchymal transition (EMT) and non-small cell lung cancer (NSCLC): a mutual association with airway disease. Med Oncol 2017; 34: 45.

Choi MJ, Cho KH, Lee S, et al. hTERT mediates norepinephrine-induced Slug expression and ovarian cancer aggressiveness. Oncogene 2015; 34: 3402-3412.

\section{Br J Cancer 2015; 113: 616-625.}

Becker-Santos DD, Guo Y, Ghaffari M, et al. Integrin-linked kinase as a target for ERG-mediated invasive properties in prostate cancer models. Carcinogenesis 2012; 33: 2558-2567.

Schlegel NC, von Planta A, Widmer DS, et al. PI3K signalling is required for a TGF $\beta$-induced epithelial-mesenchymal-like transition (EMT-like) in human melanoma cells. Exp Dermatol 2015; 24: 22-28.

Fransvea E, Mazzocca A, Santamato A, et al. Kinase activation profile associated with TGF- $\beta$-dependent migration of HCC cells: a preclinical study. Cancer Chemother Pharmacol 2011; 68: 79-86.

Feldmann G, Fendrich V, McGovern K, et al. An orally bioavailable small-molecule inhibitor of Hedgehog signaling inhibits tumor initiation and metastasis in pancreatic cancer. Mol Cancer Ther 2008; 7: 2725-2735.

Shankar S, Nall D, Tang SN, et al. Resveratrol inhibits pancreatic cancer stem cell characteristics in human and KrasG12D transgenic mice by inhibiting pluripotency maintaining factors and epithelial-mesenchymal transition. PLOS One 2011; 6: e16530.

You L, He B, Xu Z, et al. Inhibition of Wnt-2-mediated signaling induces programmed cell death in non-small-cell lung cancer cells. Oncogene 2004; 23: 6170-6174.

Konishi J, Kawaguchi KS, Vo $\mathrm{H}$, et al. $\gamma$-Secretase inhibitor prevents notch3 activation and reduces proliferation in human lung cancers. Cancer Res 2007; 67: 8051-8057.

Silverman EK. Genetics of COPD. Annu Rev Physiol 2020; 82: 413-431.

Kim WJ, Lee SD. Candidate genes for COPD: current evidence and research. Int J Chron Obstruct Pulmon Dis 2015; 10: 2249-2255.

Wang L, Zhao H, Zhang L, et al. HSP90AA1, ADRB2, TBL1XR1 and HSPB1 are chronic obstructive pulmonary disease-related genes that facilitate squamous cell lung cancer progression. Oncol Lett 2020; 19: 2115-2122.

Matsumura K, Ito S. Novel biomarker genes which distinguish between smokers and chronic obstructive pulmonary disease patients with machine learning approach. BMC Pulm Med 2020; 20: 29.

Kachroo P, Morrow JD, Kho AT, et al. Co-methylation analysis in lung tissue identifies pathways for fetal origins of COPD. Eur Respir J 2020; 56: 1902347.

Gonzalez DM, Medici D. Signaling mechanisms of the epithelial-mesenchymal transition. Sci Signal 2014; 7: re8.

Zhao R, Zhou H, Zhu J. MMP-9-C1562T polymorphism and susceptibility to chronic obstructive pulmonary disease: a meta-analysis. Medicine (Baltimore) 2020; 99: e21479.

DeVries A, Vercelli D. Of pleiotropy and trajectories: does the TGF- $\beta$ pathway link childhood asthma and chronic obstructive pulmonary disease? J Allergy Clin Immunol 2018; 141: 1992-1996.

Bowatte G, Erbas B, Lodge CJ, et al. Traffic-related air pollution exposure over a 5-year period is associated with increased risk of asthma and poor lung function in middle age. Eur Respir J 2017; 50: 1602357.

Dai X, Bui DS, Perret JL, et al. Exposure to household air pollution over 10 years is related to asthma and lung function decline. Eur Respir J 2021; 57: 2000602.

Lee J, Sandford A, Man P, et al. Is the aging process accelerated in chronic obstructive pulmonary disease? Curr Opin Pulm Med 2011; 17: 90-97.

Jin M, Lee EC, Ra SW, et al. Relationship of absolute telomere length with quality of life, exacerbations, and mortality in COPD. Chest 2018; 154: 266-273. 
van Moorsel CHM. Trade-offs in aging lung diseases: a review on shared but opposite genetic risk variants in idiopathic pulmonary fibrosis, lung cancer and chronic obstructive pulmonary disease. Curr Opin Pulm Med 2018; 24: 309-317.

87 Occhipinti M, Larici AR, Bonomo L, et al. Aging airways: between normal and disease. A multidimensional diagnostic approach by combining clinical, functional, and imaging data. Aging Dis 2017; 8: 471-485.

Bommart S, Marin G, Bourdin A, et al. Computed tomography quantification of airway remodelling in normal ageing subjects: a cross-sectional study. Eur Respir J 2015; 45: 1167-1170.

89 Telenga ED, Oudkerk M, van Ooijen PM, et al. Airway wall thickness on HRCT scans decreases with age and increases with smoking. BMC Pulm Med 2017; 17: 27.

90 Su ZQ, Guan WJ, Li SY, et al. Evaluation of the normal airway morphology using optical coherence tomography. Chest 2019; 156: 915-925.

91 Sethi S, Murphy TF. Infection in the pathogenesis and course of chronic obstructive pulmonary disease. $N$ Engl J Med 2008; 359: 2355-2365.

92 Shukla SD, Sohal SS, O'Toole RF, et al. Platelet activating factor receptor: gateway for bacterial chronic airway infection in chronic obstructive pulmonary disease and potential therapeutic target. Expert Rev Respir Med 2015; 9: 473-485.

93 Haldar K, George L, Wang Z, et al. The sputum microbiome is distinct between COPD and health, independent of smoking history. Respir Res 2020; 21: 183.

94 Bafadhel M, Haldar K, Barker B, et al. Airway bacteria measured by quantitative polymerase chain reaction and culture in patients with stable COPD: relationship with neutrophilic airway inflammation, exacerbation frequency, and lung function. Int J Chron Obstruct Pulmon Dis 2015; 10: 1075-1083.

95 Desai H, Eschberger K, Wrona C, et al. Bacterial colonisation increases daily symptoms in patients with chronic obstructive pulmonary disease. Ann Am Thorac Soc 2014; 11: 303-309.

96 Wilkinson TM, Patel IS, Wilks M, et al. Airway bacterial load and $\mathrm{FEV}_{1}$ decline in patients with chronic obstructive pulmonary disease. Am J Respir Crit Care Med 2003; 167: 1090-1095.

97 Chung KF, Adcock IM. Multifaceted mechanisms in COPD: inflammation, immunity, and tissue repair and destruction. Eur Respir J 2008; 31: 1334-1356.

98 Shukla SD, Sohal SS, Mahmood MQ, et al. Airway epithelial platelet-activating factor receptor expression is markedly upregulated in chronic obstructive pulmonary disease. Int J Chron Obstruct Pulmon Dis 2014; 9: 853-861.

99 Shukla SD, Muller HK, Latham R, et al. Platelet-activating factor receptor (PAFr) is upregulated in small airways and alveoli of smokers and COPD patients. Respirology 2016; 21: 504-510.

100 Landgraf RG, Nossi DF, Sirois P, et al. Prostaglandins, leukotrienes and PAF selectively modulate lymphocyte subset and eosinophil infiltration into the airways in a murine model of asthma. Prostaglandins Leukot Essent Fatty Acids 2007; 77: 163-172.

101 Souza DG, Pinho V, Soares AC, et al. Role of PAF receptors during intestinal ischemia and reperfusion injury. A comparative study between PAF receptor-deficient mice and PAF receptor antagonist treatment. $\mathrm{Br} J$ Pharmacol 2003; 139: 733-740.

102 Liu G, Mateer SW, Hsu A, et al. Platelet activating factor receptor regulates colitis-induced pulmonary inflammation through the NLRP3 inflammasome. Mucosal Immunol 2019; 12: 862-873.

$103 \mathrm{Kc}$ R, Shukla SD, Walters EH, et al. Temporal upregulation of host surface receptors provides a window of opportunity for bacterial adhesion and disease. Microbiology (Reading) 2017; 163: 421-430.

104 Wagner C, Goldmann T, Rohmann K, et al. Budesonide inhibits intracellular infection with non-typeable haemophilus influenzae despite its anti-inflammatory effects in respiratory cells and human lung tissue: a role for p38 MAP kinase. Respiration 2015; 90: 416-425.

105 Shukla SD, Fairbairn RL, Gell DA, et al. An antagonist of the platelet-activating factor receptor inhibits adherence of both nontypeable Haemophilus influenzae and Streptococcus pneumoniae to cultured human bronchial epithelial cells exposed to cigarette smoke. Int J Chron Obstruct Pulmon Dis 2016; 11: 1647-1655. Barbier M, Oliver A, Rao J, et al. Novel phosphorylcholine-containing protein of Pseudomonas aeruginosa chronic infection isolates interacts with airway epithelial cells. J Infect Dis 2008; 197: 465-473.

107 Weiser JN, Love JM, Moxon ER. The molecular mechanism of phase variation of $H$. influenzae lipopolysaccharide. Cell 1989; 59: 657-665.

108 McCrea KW, Xie J, Marrs CF, et al. Prevalence of genetic differences in phosphorylcholine expression between nontypeable Haemophilus influenzae and Haemophilus haemolyticus. BMC Microbiol 2010; 10: 286.

109 Shukla SD, Walters EH. COVID-19 and chronic obstructive pulmonary disease: therapeutic potential of blocking SARS-CoV2 adhesion factors. Intern Med J 2020; 50: 1153-1154.

110 Borthwick LA, Sunny SS, Oliphant V, et al. Pseudomonas aeruginosa accentuates epithelial-to-mesenchymal transition in the airway. Eur Respir J 2011; 37: 1237-1247.

111 Borthwick LA, Gardner A, De Soyza A, et al. Transforming growth factor- $\beta_{1}$ (TGF- $\left.\beta_{1}\right)$ driven epithelial to mesenchymal transition (EMT) is accentuated by tumour necrosis factor $\alpha$ (TNF $\alpha$ ) via crosstalk between the SMAD and NF-KB pathways. Cancer Microenviron 2012; 5: 45-57. 
112 Chia SB, Nolin JD, Aboushousha R, et al. Glutaredoxin deficiency promotes activation of the transforming growth factor beta pathway in airway epithelial cells, in association with fibrotic airway remodeling. Redox Biol 2020; 37: 101720.

113 Sohal SS, Mahmood MQ, Walters EH. Clinical significance of epithelial mesenchymal transition (EMT) in chronic obstructive pulmonary disease (COPD): potential target for prevention of airway fibrosis and lung cancer. Clin Transl Med 2014; 3: 33.

114 Raymakers AJN, Sadatsafavi M, Sin DD, et al. Inhaled corticosteroids and the risk of lung cancer in COPD: a population-based cohort study. Eur Respir J 2019; 53: 1801257.

115 Sohal SS, Soltani A, Reid D, et al. A randomised controlled trial of inhaled corticosteroids (ICS) on markers of epithelial-mesenchymal transition (EMT) in large airway samples in COPD: an exploratory proof of concept study. Int J Chron Obstruct Pulmon Dis 2014; 9: 533-542.

116 Milara J, Peiró T, Serrano A, et al. Roflumilast N-oxide inhibits bronchial epithelial to mesenchymal transition induced by cigarette smoke in smokers with COPD. Pulm Pharmacol Ther 2014; 28: 138-148.

117 Wang Q, Wang Y, Zhang Y, et al. The role of UPAR in epithelial-mesenchymal transition in small airway epithelium of patients with chronic obstructive pulmonary disease. Respir Res 2013; 14: 67.

118 Thomson NC. Clinical studies of statins in asthma and COPD. Curr Mol Pharmacol 2017; 10: 60-71.

119 Raymakers A, Sin DD, Sadatsafavi M, et al. Statin use and lung cancer risk in chronic obstructive pulmonary disease patients: a population-based cohort study. Respir Res 2020; 21: 118.

120 Nishikawa S, Menju T, Takahashi K, et al. Statins may have double-edged effects in patients with lung adenocarcinoma after lung resection. Cancer Manag Res 2019; 11: 3419-3432.

121 Brasier AR, Zhou J. Validation of the epigenetic reader bromodomain-containing protein 4 (BRD4) as a therapeutic target for treatment of airway remodeling. Drug Discov Today 2020; 25: 126-132.

122 Bui DS, Perret JL, Walters EH, et al. Lifetime risk factors for pre- and post-bronchodilator lung function decline. A population-based study. Ann Am Thorac Soc 2020; 17: 302-312. 